\title{
MeCAnismos de tOleRAnCia a elementos POTENCIALMENTE TÓXICOS EN PLANTAS
}

\author{
Daniel González-Mendoza ${ }^{1,2,3}$ y Omar Zapata-Pérez ${ }^{1}$ \\ 1 Departamento de Recursos del Mar, Laboratorio de Ecotoxicología, \\ Centro de Investigación y Estudios Avanzados del IPN (CINVESTAV), \\ Unidad Mérida. Km. 6, Antigua Carretera a Progreso, C.P. 97310, Mérida, Yucatán, México. \\ 2 Dirección actual: Instituto de Ciencias Agrícolas, Universidad Autónoma de Baja California. \\ Carretera a Delta s/n, C.P. 21705, Ejido Nuevo León, Baja California, México. \\ ${ }^{3}$ Autor para la correspondencia. Correo-e: daniasaf@gmail.com
}

\begin{abstract}
Resumen: Las plantas presentan una serie de mecanismos celulares que pueden estar participando en la tolerancia a elementos potencialmente tóxicos. Estos mecanismos consisten en la formación de micorrizas, el secuestro del metal en la pared celular de la planta, así como la precipitación con exudados radicales, la reducción de la absorción del metal por la membrana plasmática y la quelación del metal en el citosol por fitoquelatinas, metalotioneinas, histidina y prolina en forma libre. Asimismo, pueden involucrar el secuestro del metal en vacuolas mediante transportadores específicos localizados en el tonoplasto. Esta revisión ofrece una descripción de la participación de cada uno de los mecanismos de tolerancia en las plantas a metales potencialmente tóxicos. Palabras clave: contaminación, metales tóxicos, quelación, tolerancia, toxicidad en plantas.
\end{abstract}

\begin{abstract}
Plants possess a wide array of potential cellular mechanisms that may be involved in the tolerance to potentially toxic elements. These mechanisms include mycorrhizal associations, heavy metals binding to cell wall, precipitation by extracellular exudates; reduction in uptake or efflux pumping of metals at the plasma membrane, chelation of metals in the cytosol by peptides such as phytochelatins, metallothionein, histidina free, proline free, and the compartmentation of metals in the vacuole by tonoplast-located transporters. This review provides a broad overview of the evidence of the involvement of each mechanism in plants' tolerance to potentially toxic metals.
\end{abstract}

Key words: chelation, pollution, tolerance, toxic metals, toxicity in plants.

$\mathbf{E}^{\prime}$ ambiente terrestre se encuentra en constante cambio esto contribuye a que las fuerzas naturales de selección produzcan modificaciones en los organismos (Pollard, 2000). En el caso de las plantas, estas modificaciones les permiten adaptarse a las nuevas condiciones ambientales a través de cambios genéticos en las poblaciones durante un periodo de tiempo gobernado por la selección natural (Pollard, 2000). El proceso evolutivo puede ser analizado en dos niveles: la microevolución y la macroevolución. La microevolución consiste en cambios evolutivos producidos en las especies en periodos de tiempo relativamente cortos debido a la fuerza de la selección natural, mientras que la macroevolución involucra cambios evolutivos entre especies y se presenta generalmente después de largos periodos de tiempo (Bondada y Ma, 2002). Un ejemplo de microevolu- ción en organismos vegetales es el desarrollo de tolerancia a metales. En este proceso las actividades antropogénicas actúan como la fuerza de selección que genera los cambios evolutivos en los organismos, en periodos de tiempo relativamente cortos (cientos y décadas de años) (Bondada y Ma, 2002).

La tolerancia a elementos potencialmente tóxicos (metales esenciales y no esenciales) en los organismos vegetales puede definirse como el resultado de un proceso evolutivo que confiere a distintas especies de plantas la capacidad de crecer y desarrollarse en ambientes con concentraciones elevadas de elementos potencialmente tóxicos (Linhart y Grant 1996; Hall 2002). Los cambios evolutivos que han dado origen a la tolerancia se deben al desarrollo de una serie de mecanismos eficientes y específicos (procesos adapta- 
tivos) que permiten mantener la toma de elementos esenciales dentro de intervalos fisiológicos permisibles, además de proporcionar la capacidad de inactivar metabólicamente los elementos esenciales y no esenciales cuando representan un riesgo para la integridad celular (Linhart y Grant, 1996; Cai y Ma 2002).

Schat et al. (2000) clasificaron a la tolerancia en dos tipos de acuerdo con el conjunto de mecanismos moleculares o bioquímicos que la conforman: (1) co-tolerancia, la cual puede ser el resultado de un mecanismo específico que confiere una tolerancia a diversos metales; y (2) tolerancia múltiple, en la que la tolerancia es generada por una serie de mecanismos independientes para cada metal o metales que interactúan de manera conjunta para evitar el daño a la planta. De estas dos ideas, las evidencias sugieren que la tolerancia múltiple es el tipo que se presenta en la mayoría de las plantas (Macnair et al., 2000).

La presencia de tolerancia múltiple ha permitido que las plantas desarrollen tres estrategias básicas para establecerse en suelos con niveles tóxicos de metales: (1) exclusión: esta estrategia consiste en una limitada acumulación de metales en las partes aéreas de las plantas e involucra una acumulación preferente en el sistema radical; (2) indicadoras: esta estrategia se caracteriza porque las plantas acumulan metales en sus tejidos que generalmente reflejan los niveles de metales presentes en el suelo; y (3) hiperacumulación: esta estrategia se caracteriza por la capacidad de la planta de bioacumular altas concentraciones de metales en sus tejidos, principalmente en las partes aéreas (Baker, 1981; Baker y Walker, 1990; Vogel-Mikuš et al., 2006). Considerando el criterio de la presencia de tolerancia múltiple en las plantas, Hall (2002) divide los posibles mecanismos de tolerancia en las siguientes formas: (1) mecanismos externos de tolerancia: hongos micorrizógenos; y (2) mecanismos internos de tolerancia: (a) enlace a la pared celular y exudados radicales, (b) quelación de los metales por diversos ligandos en el citosol (fitoquelatinas, metalotioneinas y aminoácidos), (c) presencia de proteínas de estrés térmico, y (d) acumulación en la vacuola.

\section{Mecanismos externos de tolerancia}

Hongos micorrícicos (HM). En las plantas el proceso de adsorción de metales puede estar influenciado por microorganismos que están íntimamente asociados con la raíz (comunidad rizosférica). Entre estos microorganismos, los hongos micorrícicos (HM) constituyen uno de los componentes más importantes de la rizósfera, ya que forman asociaciones mutualistas (micorrizas) con diversas especies de plantas. Los HM pueden ser clasificados en dos grupos de acuerdo con el tipo de colonización de la planta hospedara: hongos micorrícicos arbusculares (HMA), los cuales se caracterizan por colonizar intracelularmente el sistema radical de $80 \%$ de las especies vegetales, y los hongos ectomicorrícicos (HEM), que se caracterizan por colonizar la raíz extra-celularmente de $3 \%$ de las especies vegetales (Shilev et al., 2001; Harrison, 2005). En el caso de los HEM, los mecanismos que se pueden presentar son: (1) movilidad del metal en el apoplasto, en donde es retenido por la red de Hartig evitando la entrada a la raíz; (2) reducción de la movilidad del metal en el apoplasto como resultado de la hidrofobicidad del hongo; (3) secreción de sustancias quelantes como ácidos orgánicos y otras sustancias producidas por el HEM; y (4) retención de los metales en el micelio externo del hongo (Jentschke y Godbold, 2000; Adriaensen et al., 2004).

En el caso de HMA los mecanismos son: (1) inmovilización extracelular de los metales por la ácidos orgánicos (AO); (2) reducción de la transferencia de iones del sistema radical al tallo, por precipitación intracelular del metal por $\mathrm{PO}^{-4}$; y (3) adsorción de los iones metálicos en la pared celular de diferentes estructuras del hongo (hifa, esporas, micelio extra radical) y la retención del metal en la micorrizósfera por la producción de proteínas específicas (p. ej. glomalina) (Zhu et al., 2001; Tullio et al., 2003; González-Chávez et al., 2004). No obstante, existen factores que pueden afectar la tolerancia a metales durante la asociación HM-planta. Entre estos factores encontramos a la especie de planta y de hongo empleado, el tipo de metal y su disponibilidad, las condiciones edáficas, las condiciones de cultivo de la planta y la densidad del sistema radical (Guo et al., 1996; del Val et al., 1999; Joner y Leyval, 2000). Esto se ha observado en plantas micorrizadas de Pinus sylvestris, en las que se genera una reducción de $\mathrm{Zn}$ en su sistema radical al ser inoculados con Paxillus involutus; no obstante, al emplear Thelephora terrestris (otra especie de HEM) se genera un efecto contrario (Colpaert y Van Assche 1992). Además, en otras especies de HEM, Suillus bovinus y T. terrestris, se ha observado que ambos organismos protegen a su hospedador del $\mathrm{Cu}$, pero varían en su capacidad de retención del metal (Van Tichelen et al., 2001).

\section{Mecanismos internos de tolerancia}

Exudados radicales. Diversos estudios han demostrado que los exudados radicales están formados principalmente por ácidos orgánicos de bajo peso molecular (AO), generados principalmente a partir del ciclo de ácido tricarboxílicos (Young et al., 1998). La importancia de los AO en la tolerancia a metales en el sistema radical se debe a que éstos pueden influir en la solubilidad de elementos esenciales y no esenciales de manera directa mediante la acidificación, la quelación, la precipitación y procesos de oxido-reducción en la rizósfera, y de forma indirecta a través de efectos en la actividad microbiana, en las propiedades físicas de la rizósfera y en la dinámica de crecimiento de la raíz (Marschner, 1995). La participación de los AO en la tolerancia a metales se observa principalmente en plantas expuestas al aluminio; así lo confirman los trabajos realizados por Zheng et al., (1998) 
quienes demostraron que al exponer plantas de Fagopyrum esculentum al Al y La observaron que la mayor producción de ácido oxálico estuvo asociada con la tolerancia al Al, al evitar su entrada a la célula. Resultados semejantes fueron indicados por Yang et al. (2005), quienes observaron un incremento del flujo de ácido oxálico al sistema radical de Spinacia oleracea L. cv. Quanneng en plantas tratadas con $\mathrm{Al}$, pero no cuando fueron expuestas a una deficiencia de fósforo y un exceso de La.

En cuanto a la participación de los $\mathrm{AO}$ en la tolerancia a otros metales, Salt et al. (2000) observaron una acumulación de citrato en los exudados radicales de plantas no hiperacumuladoras, lo que contribuye a una menor absorción de Ni durante una exposición prolongada a este metal mediante la formación y la precipitación de complejos AO-Ni en la rizósfera. No obstante, en plantas hiperacumuladoras de Cd (Thlaspi caerulescens) y Ni (Alyssum bertolonii) se ha observado que existe una respuesta contraria. Debido a la elevada presencia de ácidos cítrico, málico y oxálico en las raíces laterales, se estimula la formación de complejos de AO-metal en la rizósfera, facilitando la absorción y la retención intracelular de los metales en el sistema radical, así como el transporte a vacuolas de los tejidos por medio de proteínas de membrana del tonoplasto (Ma et al., 2005; Callahan et al., 2006).

La producción de $\mathrm{AO}$ en las plantas posiblemente se genera cuando el metal entra en contacto con el ápice de la raíz, activando el flujo de $\mathrm{AO}$, lo cual permite su ingreso a la célula por diferentes rutas (Ryan y Dehlaize, 2001). Independientemente de los pasos que siga el metal, se produce una inducción del metabolismo de AO y se lleva a cabo la protonización de los compuestos orgánicos, los cuales son enviados al citosol en donde forman complejos con los cationes no esenciales. Además, el pH citosólico se mantiene estable mediante la función coordinada de los transportadores de AO y los canales de K (Ryan y Dehlaize, 2001).

Componentes de la pared celular. La idea de que los componentes de la pared celular como la celulosa y la lignina pueden contribuir a la tolerancia a metales en las plantas fue propuesta alrededor de 1970 (Thurman, 1981). A partir de entonces se han realizado diversos estudios en los que se propone que el arreglo estructural de la celulosa y la lignina les permite formar enlaces covalentes a través de sus átomos de oxígeno con los metales, secuestrándolos en el apoplasto (Carrier et al., 2003). Este proceso fue reportado por Crist et al. (2002) y Marmiroli et al. (2005), quienes analizaron por espectroscopía de rayos $\mathrm{X}$ el proceso de acumulación de $\mathrm{Pb}$ en raíz de Medicago sativa y Juglans regia, registrando la formación de enlaces del metal con complejos de lignina y celulosa, respectivamente. Por otra parte, Ederli et al. (2004) y Wójcik y Tukiendorf, (2005) observaron que el secuestro de metales como $\mathrm{Zn}, \mathrm{Cu}$, y $\mathrm{Cd}$ en la pared celular de la raíz de Phragmites australi y Zea mays está relacionado con una mayor lignificación de las células radicales. Los autores atribuyen este efecto al incremento de la actividad de la peroxidasa que estimula la biosíntesis de la lignina en respuesta al estrés causado por estos metales.

No obstante estos resultados, la participación de la pared celular como un mecanismo de tolerancia a metales en las plantas es motivo de controversia. Por ejemplo, Ernst et al. (1992) y Hall (2002) mencionan que aún cuando la pared celular está directamente en contacto con los metales presentes en el suelo, la absorción del metal por ésta puede ser limitada. Sin embargo, esto no parece ser aplicable a las a algas, ya que para estos organismos diversos autores opinan que la retención de los metales por la pared celular parece ser uno de los mecanismos principales de tolerancia (Yun y Volesky, 2003; Padilla et al., 2005). Esta diferencia se debe a que la pared celular de las algas está conformada por numerosas capas microfibrilares de celulosa y alginatos (polímero rico en grupos sulfatos que constituye entre $20 \mathrm{y}$ $40 \%$ de la biomasa) en forma de geles, lo cual le confiere una alta capacidad de bioadsorción de metales (Lodeiro et al., 2005).

\section{Quelación de los metales en el citosol por diversos ligan- dos}

La quelación se puede definir como la capacidad de una molécula para formar un complejo con un metal y así formar un nuevo compuesto con propiedades químicas diferentes del original. La quelación es un proceso homeostático en el que participan dos tipos de moléculas: (1) moléculas transportadoras, encargadas de transferir iones específicos de metales a organelos particulares como las vacuolas, los cloroplastos y las mitocondrias que los requieran; y (2) los ligandos de alta afinidad como las fitoquelatinas, las metalotioneinas, los ácidos orgánicos, las proteínas de estrés térmico y los aminoácidos, los cuales contribuyen a la desintoxicación a la vez que mantienen estable la concentración de iones de metales en el citosol (Clemens, 2001; Hall, 2002).

Fitoquelatinas (FQS). Los ligandos de alta afinidad mejor caracterizados en las células de las plantas son las fitoquelatinas (FQS) y las metalotioneinas (MTs) (Zenk, 1996). Las FQS han sido identificadas en una amplia variedad de plantas, en ciertos microorganismos y en hongos (Rauser, 1995; Cobbet y Goldsbrough, 2002). En las plantas, las fitoquelatinas son péptidos con la fórmula general ( $\gamma$-Glu-Cys) -Gly $(\mathrm{n}=2-11)$, en donde las estructuras ( $\gamma$-Glu-Cys) $)_{2}$-Gly $\left(\mathrm{FQ}_{2}\right)$ y $(\gamma \text {-Glu-Cys) })_{3}$-Gly $\left(\mathrm{FQ}_{3}\right.$ ) son las más comunes (Cobbett y Goldsbrough, 2002). Las FQS fueron detectadas en primera instancia por el grupo de Hayashi, quienes observaron la formación de complejos de péptidos con iones de cadmio en la levadura Schizosaccharomyces pompe, a las cuales denominaron 'cadistinas' (Murasugi et al., 1981). Por otro lado, Grill et al. (1986) observaron la presencia de los péptidos 
con alto grado de polimerización en varios cultivos celulares de plantas expuestas a iones de cadmio, a los cuales denominaron 'fitoquelatinas'.

La síntesis de las FQS en las plantas se realiza vía enzimática a partir de la $\gamma$-glutamilcisteina dipeptidil transpeptidasa, comúnmente denominada fitoquelatina sintetasa, la cual se expresa constitutivamente pero también puede ser regulada a nivel de transcripción y postranscripción (Heiss et al., 2003). La FQS tiene como sustrato al glutatión (GSH), cuya síntesis puede ser afectada por el estrés oxidativo y es producido por dos reacciones catalizadas por la $\gamma$-glutamilcistina sintetasa (EC 6.3.2.2) y la GSH sintetasa (EC 6.3.2.3) a partir de la glutamina (Glu), la cisteína (Cys) y la glicina (Gly) (Inouhe, 2005).

El mecanismo de acción de las FQS en las plantas consiste en la formación de complejos no tóxicos con iones de metales mediante la interacción con los grupos tioles de cisteína (Cys), formando un complejo FQ-metal de bajo peso molecular (CBPM), el cual posteriormente se une a iones sulfuro ( $\mathrm{S}^{2-}$ ) en el citosol, estabilizando al CBPM, y forma moléculas complejas de alto peso molecular (CAPM) (Hirata et al., 2005). Posteriormente, estas moléculas atraviesan el tonoplasto, posiblemente usando transportadores $\mathrm{ABC}$; una vez dentro de la vacuola, los ácidos orgánicos presentes (malato, citrato, oxalato) retienen a los iones de los metales y disocian el complejo FQ-metal (Salt y Rauser,1995). Todavía no está claro qué le ocurre a las FQS una vez separada del metal; sin embargo, Gadapati y Macfie (2006) sugieren que posiblemente las FQS pueden ser degradadas en la vacuola o bien retornadas al citoplasma para formar nuevos complejos con los iones de los metales.

En organismos vegetales acuáticos y terrestres, existe evidencia que muestra que las FQS desempeñan una importante función en la desintoxicación de diferentes metales (Cobbett y Goldsbrough, 2000), debido a que las concentraciones elevadas de distintos iones pueden estimular su síntesis; las FQS secuestran a los metales (esenciales y no esenciales) y los distribuyen preferentemente a la vacuola de los distintos tejidos (Mishra et al., 2006; Perales-Vela et al., 2006). No obstante, su papel en la tolerancia no está claro y puede variar entre las especies de organismos vegetales y el tipo de metal, de acuerdo con los trabajos de Schat et al. (2002) y Ebbs et al.(2002), quienes encontraron que al inhibir la actividad de la enzima FQsintasa (clave para la síntesis de $\mathrm{FQ}$ ) en plantas tolerantes a $\mathrm{Co}, \mathrm{Ni}, \mathrm{Cu}$ y $\mathrm{Zn}$, no se observaron variaciones en su capacidad de tolerancia, lo cual hace suponer la participación de otros mecanismos en el proceso de regulación homeostática. Por otra parte, Tsuji et al. (2002) han sugerido que las FQS no sólo desempeñan un papel importante en la destoxificación de metales, sino que además participan en la mitigación del estrés oxidativo. Por ejemplo, al experimentar con extractos celulares de Phaeodactylum tricornutum y Allium sativum, respectivamente, Morelli y Scarano (2004) y Zhang et al. (2005) observaron que la exposición a Cu estimuló la presencia de FQS libres de metales. Por esta razón, estos autores suponen que las FQS pueden estar en forma oxidada y por lo tanto participar en la disminución de especies reactivas de oxígeno.

Metalotioneinas. Las metalotioneinas (MTS) son proteínas de bajo peso molecular (4-8 kDa), generadas transcripcionalmente y con capacidad de ligar iones metálicos (por su alto contenido en residuos de cisteína, 30\% del total de la proteína) para evitar así una intoxicación por metales. Su descubrimiento tiene alrededor de 40 años y se describieron por primera vez como un tipo de proteína quelatante del $\mathrm{Cd}$ en el hígado de caballo. Los genes que codifican para las MTS han sido detectados en animales (vertebrados e invertebrados), plantas y algunos procariontes (Cobbett y Goldsbrough, 2002). Las MTS se clasifican en dos clases, considerando las secuencias de aminoácidos que las conforman. La clase MT1 incluye únicamente a las MTs de mamíferos (Klassen et al., 1999), mientras que la clase MT2 incluye a las MTs de hongos, invertebrados y plantas (Robinson et al., 1993). Para comprender la regulación de las MTs se ha estudiado en algunas plantas la expresión de sus genes mediante el uso de técnicas de biología molecular (Guo et al., 2003).

Las MTs de clase II presentes en las plantas se agrupan en cuatro tipos (Mt1, Mt2, Mt3, Mt4) de acuerdo con el ordenamiento de los residuos de cisteína en la proteína, y fueron identificadas por primera vez en embriones maduros de trigo como una proteína 'quelatante' del Zn (Cobbett y Goldsbrough, 2002). Desde el descubrimiento de las MTS en las plantas, su estudio se ha enfocado en evaluar la expresión de genes en diferentes etapas del desarrollo de las plantas terrestres, principalmente en angiospermas, grupo en el que se ha encontrado que la expresión de las MTS puede estar restringida en diferentes tejidos de las plantas. Por ejemplo, la presencia de Mt4 fue identificada en semillas de maíz como un posible mecanismo para proporcionar los micronutrientes requeridos durante la germinación (White y Rivin, 1995).

En el caso de la MT1, se observó una mayor inducción del ARN mensajero (ARNm) en raíces de Arabidopsis thaliana (Zhou y Goldsbrough, 1995), mientras que la expresión del gen MT2 en la misma planta presentó una mayor inducción del ARNm en el tallo y en las hojas que en raíces (Guo et al., 2003). Para el caso del gen de la MT3, se observó que existió una sobreexpresión en las hojas de las plantas como Arabidopsis thaliana y en plantas como el guineo (plátano, banano), el kiwi y la manzana (Ledger y Gardner, 1994; Guo et al., 2003).

El mecanismo de acción de las MTs en la regulación homeostática y la tolerancia a los metales en las plantas no está completamente establecido. Sin embargo, se ha reconocido su participación en la tolerancia a metales en diferentes especies de plantas, como es el caso de Oryza sativa y Arabidopsis thaliana al ser tratadas con $\mathrm{Cu}, \mathrm{Cd}$ y $\mathrm{Zn}$, especies en 
las que se observó una mayor expresión de ARNm de MT en las plantas tratadas con $\mathrm{Cu}$ (Hsieh et al., 1996).

No obstante, al exponer a Brassica juncea y Vicia faba a $\mathrm{Cu}$ y Zn, la expresión del gen de la Mt1 en el tallo de estas plantas fue inhibida con el tratamiento de $\mathrm{Cu}$ (Foley et al., 1997; Schafer et al., 1997). Asimismo, se demostró que la expresión del gen Mt2 tuvo una asociación positiva con la supervivencia de plantas de Arabidopsis y Silene vulgaris expuestas a Cu (Murphy y Taiz, 1995; van Hoof Nalm et al., 2001). Además, se ha descrito la participación de los genes de las MTs en la senescencia de las hojas, proceso en el que se ha observado que los niveles de ARNm de Mt2 se incrementan en las hojas senescentes de arroz y Arabidopsis, lo cual ha sido relacionado con la protección de las células contra el estrés oxidativo (Hsieh et al., 1995; García-Hernández et al., 1998).

Histidina. La histidina (Hi) es un aminoácido libre que desempeña una función importante en la tolerancia a metales en plantas hiperacumuladoras, ya que puede secuestrar al metal mediante enlaces con sus grupos carboxilato, amino e imadazol (Krämer et al., 1996). La formación de complejos de Hi-metal ha sido observada en raíces de plantas hiperacumuladoras como Alyssum lesbiacum expuestas principalmente a $\mathrm{Ni}$; en este caso, la $\mathrm{Hi}$ puede actuar quelando al metal, facilitando así el transporte del metal de la raíz al tallo vía xilema y su posterior acumulación en vacuola por los AO (Kerkeb y Krämer, 2003). La regulación de la biosíntesis de la Hi en plantas hiperacumuladoras no está completamente comprendida, pero diversos autores han encontrado que las concentraciones de $\mathrm{Hi}$ en raíces de plantas hiperacumuladoras de $\mathrm{Ni}$ (e.g. Alyssum lesbiacum) son mayores que en las plantas no-hiperacumuladoras (e.g. Brassica juncea) (Kerkeb y Krämer, 2003). Resultados similares han sido indicados por Persans et al. (2001) en las raíces de Thlaspi goesingense (planta hiperacumuladora de $\mathrm{Ni}$ ), las cuales muestran concentraciones de Hi hasta 17 veces mayores las de $T$. arvense (planta no-hiperacumuladora).

En una investigación reciente en donde se utilizó una enzima clave en la biosíntesis de $\mathrm{Hi}$, la fosforibosiltransferasa-ATP (ATP-PRT), Ingle et al. (2005) encontraron que el incremento en la abundancia de transcriptos de la ATP-PRT en plantas de A. serpyllifolium y A. lesbiacum estimulaban una mayor concentración de Hi y una mayor acumulación de Ni en los diferentes tejidos de la planta. Estos resultados sugieren que la expresión del gen ATP-PRT desempeña una función importante en la regulación de la Hi libre, contribuyendo así a la tolerancia al Ni en las especies de Alyssum y posiblemente en otras especies tolerantes a este metal.

Prolina. La prolina es un aminoácido que en su forma libre participa en la destoxificación de metales en la planta (Mehta y Gaur, 1999). Algunos autores han sugerido que puede actuar como osmoprotector o como inhibidor de la lipo-pe- roxidación, actuando en el secuestro de especies reactivas de oxígeno (Alia et al., 2001). Estudios recientes realizados por distintos grupos de investigación han confirmado que el aporte de la prolina a la tolerancia a metales en las plantas se lleva a cabo mediante su participación como agente antioxidante.

Por ejemplo, Siripornadulsil et al. (2002) observaron que la prolina libre puede actuar como antioxidante en células de Chlamydomonas reinhardtii (microalga) tratadas con $\mathrm{Cd}$, evitando que las especies reactivas de oxígeno reaccionaran con el glutatión; esto permite mantener estables los niveles de glutatión en el citoplasma, facilitando la síntesis de fitoquelatinas, las cuales incrementaron la tolerancia de este organismos al metal. Esto fue confirmado por Yonamine et al. (2004) en cultivos celulares de Nicotiana tabacum, en donde al estimular la inducción temporal del gen NtHAL3a se generó una mayor biosíntesis de prolina libre, que disminuyó la presencia de especies reactivas de oxígeno, reflejándose en un incremento en la tolerancia de las células al Li.

Proteínas de estrés térmico. En las plantas, las proteínas de estrés térmico (HSPs) se localizan en diferentes compartimentos celulares y se clasifican en seis clases diferentes de acuerdo con su localización: en el citosol y el núcleo (clase 1, 2 y 3 ), en los cloroplastos, en la mitocondria o asociados con el retículo endoplasmático (Sun et al., 2002; Leone et al., 2003). La función de las HSPs in vivo no ha sido comprendida por completo, pero se piensa que las HSPs del citosol pueden actuar protegiendo a las células del efecto tóxico o letal del calor, impidiendo la desnaturalización de la proteína durante el estrés y facilitando su reactivación posterior (Lee et al., 1997). No obstante, también se ha demostrado su función como chaperonas en experimentos in vivo e in vitro (Löw et al., 2000; Scharf et al., 2001).

En cuanto a la participación de estas proteínas en la tolerancia a metales, Heckathorn et al. (2004) observaron que el efecto del $\mathrm{Pb}$ y el $\mathrm{Ni}$ en cloroplastos aislados de dos variedades de Agrostis stolonifera produjeron un mayor contenido de proteína HSP en cloroplastos de la variedad tolerante, generando una mayor protección del sistema fotosintético. Según el autor, estos resultados muestran una función específica de protección por las HSPs al estrés causado por metales en esta planta. Por otra parte, Gulli et al. (2005) encontraron que al exponer plantas de Hordeum vulgare y Zea mays a Cd se generaba una mayor inducción del gen Hvhsp 17, localizado en el citoplasma, lo cual fue relacionado con la protección y reparación de proteínas durante la exposición al metal.

En cuanto a la síntesis de proteínas HSPs en plantas expuestas a metales, se ha propuesto que puede ser causado por el estrés oxidativo generado por los metales, debido a que existe una relación entre el calor y la presencia de especies reactivas (Larkindale y Knight, 2002; Gulen y Eris, 2003). Esto ha sido confirmado por Dat et al. (2000), quie- 
nes observaron que especies reactivas de oxígeno inducían termo-tolerancia en las plantas, pero es el trabajo de Vallelian-Bindschedler et al. (1998) donde se confirma esta idea, al demostrar que la generación de especies reactivas de oxígeno en el organismo producen pulsos de calor que estimulan la producción de las proteínas HSPs en las células.

Almacenamiento de metales en vacuola. La vacuola ha sido descrita como un componente celular que ocupa cerca de $80 \%$ del volumen de la célula, aunque también puede haber vacuolas de menor tamaño, las cuales pueden distinguirse por el contenido de proteínas solubles y por su proteína de membrana (acuaporinas) (Martinoia et al., 2000). Las vacuolas sirven como reservorio de metabolitos y nutrientes, y participan en el proceso de regulación homeostática del citosol (Martinoia et al., 2000). La importancia de la vacuola en la tolerancia a metales ha sido demostrada en diversos trabajos (ver revisión de De, 2000). Por ejemplo, Davies et al. (1991) observaron que existe una correlación entre la tolerancia y una mayor vacuolización de las células del meristemo de la raíz en plantas de Festuca rubra al ser expuestas a Zn. Posteriormente, en estudios de absorción de metales usando $\mathrm{Zn}^{65}$ en el tejido foliar de plantas de Hordeum vulgare, Brune et al. (1994) sugirieron que el almacenamiento rápido del $\mathrm{Zn}$ en la vacuola es un mecanismo importante para evitar el daño en el tejido por concentraciones altas del metal. También se ha demostrado que el tonoplasto es un factor clave en la tolerancia a metales, como lo confirman los trabajos de Verkleij et al. (1998), quienes al exponer al Zn, los tonoplastos aislados de plantas tolerantes y no tolerantes al metal observaron que el transporte a través del tonoplasto por proteínas de membrana a la vacuola fue 2.5 veces mayor en las plantas tolerantes, confirmando la importancia de la vacuola en la tolerancia a metales en las plantas.

\section{Conclusiones}

El incremento de residuos con elementos potencialmente tóxicos en el ambiente es un factor importante que ha ocasionado que las plantas desarrollen de una forma más rápida una amplia gama de mecanismos para poder crecer en ambientes con elevadas concentraciones de elementos esenciales y no esenciales. La prevalencia de un mecanismo $\mathrm{u}$ otro en la planta aún no está del todo comprendido. Por lo tanto, es necesario que en el futuro se lleven a cabo estudios que involucren la aplicación de herramientas moleculares y bioquímicas, así como de plantas modelo como Arabidopsis o Thlaspi caeroleus, con el fin de entender con mayor claridad el modo de acción de los mecanismos de tolerancia en el mantenimiento homeostático de la célula vegetal.

En esta revisión se muestra la importancia de conocer las bases bioquímicas y moleculares de los diferentes mecanismos de tolerancia que las plantas han desarrollado para sobrevivir en ambientes con elevadas concentraciones de elementos potencialmente tóxicos. Este tipo de conocimiento permitirá proponer soluciones a los problemas de la contaminación y la posibilidad de recuperación de los diferentes ecosistemas impactados por metales.

\section{Literatura citada}

Adriaensen K., van der Lelie D., Van Laere A., Vangronsveld J. y Colpaert J.V. 2004. A zinc-adapted fungus protects pines from zinc stress. New Phytologist 161:549-555.

Alia M.P., Mo Hanty P. y Matysik J. 2001. Effect of proline on the production of singlet oxygen. Amino Acids 21:195-200.

Baker A.M. 1981. Accumulators and excluders: strategies in the response of plants to heavy metals. Journal of Plant Nutrition 3:643-654.

Baker A.J.M. y Walker P.L. 1990. Ecophysiology of metal uptake by tolerant plants. En Shaw A.J. Ed. Heavy Metal Tolerance in Plants: Evolutionary Aspects. pp.155-177, CRC Press, Boca Raton, Florida.

Bondada B. y Ma L.Q. 2002. Tolerance of heavy metals in vascular plants: arsenic hyperaccumulation by Chinese brake fern (Pteris vittata L.). En: Chandra S. y Srivastava M. Eds. Pteridology in the New Millennium, pp. 397-420, Kluwer, Dordrecht.

Brune A., Urbach W. y Dietz K.J. 1994. Compartmentation and transport of zinc in barley primary leaves as basic mechanisms involved in zinc tolerance. Plant, Cell and Environment 17:153-162

Cai Y. y Ma L.Q. 2002. Metal tolerance, accumulation and detoxification in plants with emphasis on arsenic in terrestrial plants. En: Cai Y. y Braids O. Eds. Biogeochemistry of Environmentally Important Trace Elements, pp. 95-114, Oxford University Press, Oxford.

Callahan D.L., Baker J.M., Kolev D. y Wedd G. 2006. Metal ion ligands in hyperaccumulating plants. Journal of Biological Inorganic Chemistry 11:2-12.

Carrier P., Baryla A. y Havaux M. 2003. Cadmium distribution and microlocalization in oilseed rape (Brassica napus) after long-term growth on cadmium-contaminated soil. Planta 216: 939-950.

Cobbett C. y Goldsbrough P. 2002. Phytochelatins and metallothioneins: roles in heavy metal detoxification and homeostasis. Annual Review of Plant Biology 53:159-182.

Colpaert, J.V. y Van Assche, J.A. 1992 The effects of cadmium and cadmium-zinc interaction on the axenic growth of ectomycorrhizal fungi. Plant and Soil 145:237-243.

Clemens S. 2001. Molecular mechanisms of plant metal tolerance and homesostasis. Planta 212:475-486.

Crist R.H., Martin J.R. y Crist D.R. 2002. Heavy metal uptake by lignin: comparison of biotic ligand models with an ion-exchange process. Environmental Science and Technology 36:1485-1490.

Davies K.L, Davies M.S. y Francis D. 1991. Zinc-induced vacuolation in root meristematic cells of Festuca rubra L. Plant, Cell and Environment 14:399-406.

Dat J., Vandenbeele S., Vranová E., Van Montagu M., Inze D. y Van Breusegm F. 2000. Dual action of the active oxygen species during plant stress responses. Cellular and Molecular Life Sciences 57:779-795.

De D.N. 2000. Plant Cell Vacuoles. CSIRO Publishing. Collingwood, Australia. 
del Val C., Barea J.M. y Azcón-Aguilar C. 1999. Assessing tolerance to heavy metals of arbuscular mycorrhizal fungi isolated from sewage sludge-contaminated soils. Applied Soil Ecology 11:261-269

Ebbs S., Lau I., Ahner B. y Kochian L. 2002. Phytochelatin synthesis is not responsible for $\mathrm{Cd}$ tolerance in the $\mathrm{Zn} / \mathrm{Cd}$ hyperaccumulator Thlaspi caerulescenes (J. \& C. Presl). Planta 214:635-640.

Ederli L., Reale L., Ferranti F. y Pasqualini S. 2004. Responses induced by high concentration of cadmium in Phragmites australis roots. Physiologia Plantarum 121:66-74.

Ernst W.H.O., Verkleij J.A.C. y Schat H. 1992. Metal tolerance in plants. Acta Botanica Neerlandica 41:229-248.

Foley R.C., Liang Z.M. y Singh K.B. 1997. Analysis of type 1 metallothionein cDNAs in Vicia faba. Plant Molecular Biology 33:583-591.

Gadapati, W.R. y Macfie S.M. 2006. Phytochelatins are only partially correlated with Cd-stress in two species of Brassica. Plant Science 170:471-480.

García-Hernández M., Murphy A. y Taiz L. 1998. Metallothionein 1 and 2 have distinct but overlapping expression patterns in Arabidopsis. Plant Physiology 118:387-397.

González-Chávez M.C., Carrillo-González R., Wright S.F. y Nichols K.A. 2004. The role of glomalin, a protein produced by arbuscular mycorrhizal fungi in sequestering potentially toxic elements. Environmental Pollution 130:317-323.

Guo W.J., Bundithya W. y Goldsbrough P.B. 2003. Characterization of the Arabidopsis metallothionein gene family: tissuespecific expression and induction during senescence and in response to copper. New Phytologist 159:369-381.

Guo Y., George E. y Marschner H. 1996. Contribution of an arbuscular mycorrhizal fungus to the uptake of cadmium and nickel in bean and maize plants. Plant and Soil 184:195-205.

Gulen H. y Eris A. 2003. Some physiological changes in strawberry (Fragaria $\times$ ananassa 'Camarosa') plants under heat stress. Journal of Horticulture Science and Biotechology 78:894-898.

Gulli M., Rampino P., Lupotto E., Marmiroli N. y Perrotta C. 2005. The effect of heat stress and cadmium ions on the expression of a small hsp gene in barley and maize. Journal of Cereal Science 42:25-31.

Grill E., Winnacker E.L. y Zenk M.H. 1986. Synthesis of seven different homologous phytochelatins in metal-exposed Schizosaccharomyces pombe cells. FEBS Letters 197:115-120.

Hall J.L. 2002. Cellular mechanisms for heavy metal detoxification and tolerance. Journal of Experimental Botany 53:1-11.

Harrison M.J. 2005. Signaling in the arbuscular mycorrhizal symbiosis. Annual Review of Microbiology 59:19-42.

Heckathorn S.A., Mueller J.K., LaGuidice S., Zhu B., Barrett T., Blair B. y Dong Y. 2004. Chloroplast small heat-shock proteins protect photosynthesis during heavy metal stress. American Journal of Botany 91:1312-1318.

Heiss S., Wachter A., Bogs J., Cobbett C. y Rausch T. 2003. Phytochelatin synthase (PCS) protein is induced in Brassica juncea leaves after prolonged Cd exposure. Journal of Experimental Botany 54:1833-1839.

Hirata K., Tsuji N. y Miyamoto K. 2005. Biosynthetic regulation of phytochelatins, heavy metal-binding peptides. Journal of Bioscience and Bioengineering 100:593-599.

Hsieh H.M., Liu W.K., Chang A. y Huang P.C. 1996. RNA expression patterns of a type 2 metallothionein-like gene from rice. Plant Molecular Biology 32:525-529.
Inouhe M. 2005. Phytochelatins. Brazilian Journal of Plant Physiology 17:65-78.

Ingle R.A., Mugford S.T., Rees J.D., Campbell M.M. y Smith J.A.C. 2005. Constitutively high expression of the histidine biosynthetic pathway contributes to nickel tolerance in hyperaccumulator plants. Plant and Cell 17:2089-2106.

Jentschke G. y Godbold D.L. 2000. Metal toxicity and ectomycorrhizas. Physiologia Plantarum 109:107-116.

Joner E.J., Briones R. y Leyval C. 2000. Metal binding capacity of arbuscular mycorrhizal mycelium. Biology and Fertility of Soils 226:227-234.

Kerkeb L. y Kramer U. 2003. The role of free histidine in xylem loading of nickel in Alyssum lesbiacum and Brassica juncea. Plant Physiology 131:716-724

Klaassen C.D., Liu J. y Choudhuri S. 1999. Metallothionein: an intracellular protein to protect against cadmium toxicity. Annual Review of Pharmacology and Toxicology 39:267-294.

Krämer U., Cotter-Howells J.D., Charnock J.M., Baker A.J.M. y Smith J.A.C. 1996. Free histidine as a metal chelator in plants that accumulate nickel. Nature 379:635-638

Larkindale J. y Knight M.R. 2002. Protection against heat stressinduced oxidative damage in Arabidopsis involves calcium, abscisic acid, ethylene, and salicylic acid. Plant Physiology 128:682-695.

Lee G.J., Roseman A.M., Saibil H.R. y Vierling E. 1997. A small heat shock protein stably binds heat-denatured model substrates and can maintain a substrate in a folding-competent state. EMBO Journal 16:659-671.

Ledger S.E. y Gardner R.C. 1994. Cloning and characterization of five cDNAs for genes differentially expressed during fruit development of kiwi-fruit (Actinidia deliciosa var. deliciosa). Plant Molecular Biology 25:877-886.

Leone, A., Perrotta C. y Maresca B. 2003. Plant tolerance to heat stress: current strategies and new emergent insights. En: Di Toppi L.S. y Pawlik-Skowronska B. Eds. Abiotic Stresses in Plants, pp. 1-22. Kluwer, Dordrecht.

Linhart Y.B. y Grant M.C. 1996. Evolutionary significance of loca genetic differentiation in plants. Annual Review of Ecology and Systematics 27:237-277.

Lodeiro P., Cordero B., Barriada J.L., Herrero R. y de Vicente M.E.S. 2005. Biosorption of cadmium by biomass of brown marine macroalgae. Bioresource Technology 96:1796-1803.

Löw D., Brändle K., Nover L. y Forreiter C. 2000. Cytosolic heatstress proteins Hsp17.7 class I and Hsp17.3 class II of tomato act as molecular chaperones in vivo. Planta 211:575-582.

Ma J.F., Ueno D., Zhao F.J. y McGrath S.P. 2005. Subcellular localisation of $\mathrm{Cd}$ and $\mathrm{Zn}$ in the leaves of a Cd-hyperaccumulating ecotype of Thlaspi caerulescens. Planta 220:731-736.

Marschner H. 1995. Mineral Nutrition in Higher Plants, 2a ed, Academic Press, Londres.

Marmiroli M., Antonioli G., Maestri E. y Marmiroli N. 2005. Evidence of the involvement of plant ligno-cellulosic structure in the sequestration of $\mathrm{Pb}$ : an X-ray spectroscopy-based analysis. Environmental Pollution 134:217-227.

Macnair M.R., Tilstone G.H. y Smith S.E. 2000. The genetics of metal tolerance and accumulation in higher plants. En: Terry $\mathrm{N}$ y Banuelos G .Eds. Phytoremediation of Contaminated Soil and Water, pp. 235-250, CRC Press, Boca Raton, Florida

Martinoia E., Klein M., Geisler M., Sánchez-Fernández R. y Rea P.A. 2000. Vacuolar transport of secondary metabolites and xe- 
nobiotics. En: Robinson D. y Rogers J. Eds. Vacuolar Compartments, pp. 221-253, Sheffield Academic Press, Sheffield.

Mehta S.K. y Gaur J.P. 1999. Heavy metal-induced proline accumulation and its role in ameliorating metal toxicity in Chlorella vulgaris. New Phytologist 143:253-259.

Mishra S., Srivastava S., Tripathi R.D., Govindarajan R., Kuriakose S.V. y Prasad M.N.V. 2006. Phytochelatin synthesis and response of antioxidants during cadmium stress in Bacopa monnieri L. Plant Physiology and Biochemistry 44:25-37.

Morelli E. y Scarano G. 2004. Copper-induced changes of nonprotein thiols and antioxidant enzymes in the marine microalga Phaeodactylum tricornutum. Plant Science 167:289-296.

Murphy A. y Taiz L. 1996. Purification of copper-binding metallothionein-like proteins from Arabidopsis thaliana. Plant Physiology 111:553-553.

Murasugi A., Wada C. y Hayashi Y. 1981. Cadmium binding peptide in fission yeast, Schizosaccharomyces pombe. Journal of Biochemistry 90:1561-1564.

Padilha F.P., de Franca F.P. y da Costa A.C.A. 2005. The use of waste biomass of Sargassum sp. for the biosorption of copper from simulated semiconductor effluents. Bioresource Techno$\log y$ 96:1511-1517.

Persans M.W., Nieman K. y Salt D.E. 2001. Functional activity and role of cation-efflux family members in Ni hyperaccumulation in Thlaspi goesingense. Proceedings of the National Academy of Sciences of the United States of America 98:9995-10000.

Perales-Vela H.V., Peña-Castro J.M. y Cañizares-Villanueva R.O. 2006. Heavy metal detoxification in eukaryotic microalgae. Chemosphere 64:1-10.

Pollard A.J. 2000. Metal hyperaccumulation: a model system for coevolutionary studies. New Phytologist 146:179-181.

Rauser W.E. 1995. Phytochelatins and related peptides- structure, biosynthesis and function. Plant Physiology 109:1141-1149.

Robinson N.J., Tommey A.M. Kuske C. y Jackson P.J. 1993. Plant metallothioneins. Biochemical Journal 295:1-10.

Ryan P.R. y Delhaize E., Jones D.L. 2001. Function and mechanism of organic acid exudation from roots. Annual Review of Plant Physiology and Plant Molecular Biology 52:527-560.

Salt D.E, Kato N., Krämer U., Smith R.D. y Raskin I. 2000. The role of root exudates in nickel hyperaccumulation and tolerance in accumulator and non accumulator species of Thlaspi. En: Terry N. y Bañuelos G.S. Eds. Phytoremediation of Contaminated Soil and Water, pp. 189-200, CRC Press, Boca Raton, Florida.

Salt D.E. y Rauser W.E. 1995. MgATP-dependent transport of phytochelatins across the tonoplast of oat roots. Plant Physiology 107:1293-1301.

Schat H., Llugany M., Vooijs R., Hartley-Whitaker J. y Bleeker P.M. 2002. The role of phytochelatins in constitutive and adaptive heavy metal tolerances in hyperaccumulator and non-hyperaccumulator metallophytes. Journal of Experimental Botany 53:2381-2392.

Schat H., Llugany M. y Bernhard R. 2000. Metal-specific patterns of tolerance, uptake and transport of heavy metals in hyperaccumulating and nonhyperaccumulating metallophytes. En: Terry N. y Banuelos G. Eds. Phytoremediation of Contaminated Soil and Water, pp. 171-188. CRC Press, Boca Raton, Florida.

Shilev S.I., Ruso J., Puig A., Benlloch M., Jorrin J. y Sancho E. 2001. Rhizospheric bacteria promote sunflower (Helianthus annus L.) plant growth and tolerance to heavy metals. Minerva Biotecnologica 13:37-39.
Scharf K.D., Siddique M. y Vierling E. 2001. The expanding family of Arabidopsis thaliana small heat stress proteins and a new family of proteins containing $\alpha$-crystallin domains (Acd proteins). Cell Stress and Chaperones 6:225-237.

Schafer H.J., Greiner S., Rausch T. y HaagKerwer A. 1997. In seedlings of the heavy metal accumulator Brassica juncea $\mathrm{Cu}^{2+}$ differentially affects transcript amounts for $\gamma$-glutamylcysteine synthetase $(\gamma$-ECS) and metallothionein (MT2). FEBS Letters 404:216-220.

Siripornadulsil S., Traina S., Verma D.P.S. y Sayre R.T. 2002. Molecular mechanisms of proline-mediated tolerance to toxic heavy metals in transgenic microalgae. Plant and Cell 14:28372847.

Sun W.N., van Montagu M. y Verbruggen N. 2002. Small heat shock proteins and stress tolerance in plants. Biochimica et Biophysica Acta-Gene Structure and Expression 1577:1-9.

Tullio M., Pierandrei F., Salermo A. y Rea E. 2003. Tolerance to cadmium of vesicular arbuscular mycorrhizae spores isolated from a cadmium-polluted and unpolluted soil. Biology and Fertility of Soils 37:211-214.

Thurman D.A. 1981. Metals in the environment. En: Lepp N.W. Ed. Effect of Heavy Metal Pollution on Plants, Volume 2, Metals in the Environment, pp. 239-247, Applied Science Publishers, Englewood, Nueva Jersey.

Tsuji N., Hirayanagi N., Okada M., Miyasaka H., Hirata K., Zenk M.H. y Miyamoto K. 2002. Enhancement of tolerance to heavy metals and oxidative stress in Dunaliella tertiolecta by $\mathrm{Zn}$-induced phytochelatin synthesis. Biochemical and Biophysical Research Communications 293:653-659.

van Hoof N.A.L.M., Hassinen V.H., Hakvoort H.W.J., Ballintijn K.F., Schat H., Verkleij J.A.C., Ernst W.H.O., Karenlampi S.O. y Tervahauta A.I. 2001. Enhanced copper tolerance in Silene vulgaris (Moench) Garcke populations from copper mines is associated with increased transcript levels of a 2b-type metallothionein gene. Plant Physiology 126:1519-1526.

Van Tichelen, K.K., Colpaert J.V. y Vangronsveld J. 2001. Ectomycorrhizal protection of Pinus sylvestris against copper toxicity. New Phytologist 150:203-213.

Vallelian-Bindschedler L., Schweizer P., Mosinger E. y Metraux J.P. 1998. Heat-induced resistance in barley to powdery mildew (Blumeria graminis f. sp. hordei) is associated with a burst of active oxygen species. Physiological and Molecular Plant Pathology 52:185-199.

Verkleij J.A.C., Koevoets P.L.M., Blake-Kalff M.M.A. y Chardonnens A.N. 1998. Evidence for an important role of the tonoplast in the mechanism of naturally selected zinc tolerance in Silene vulgaris. Journal of Plant Physiology 153:188-191.

Vogel-Mikuš K., Pongrac P., Kump P., Nečemer M. y Regvar M. 2006. Colonisation of a $\mathrm{Zn}, \mathrm{Cd}$ and $\mathrm{Pb}$ hyperaccumulator Thlaspi praecox Wulfen with indigenous arbuscular mycorrhizal fungal mixture induces changes in heavy metal and nutrient uptake. Environmental Pollution 139:362-371.

White C.N. y Rivin C.J. 1995. Characterization and expression of a cDNA encoding a seed-specific metallothionein in maize. Plant Physiology 108:831-832.

Wójcik M. y Tukiendorf A. 2005. Cadmium uptake, localization and detoxification in Zea mays. Biologia Plantarum 49:237245.

Yang J.L., Zheng S.J., He Y.F. y Matsumoto H. 2005. Aluminum resistance requires resistance to acid stress: a case study with 
spinach that exudes oxalate rapidly when exposed to $\mathrm{Al}$ stress. Journal of Experimental Botany 56:1197-1203.

Yonamine I., Yoshida K., Kido K., Nakagawa A., Nakayama H. y Shinmyo A. 2004. Overexpression of NtHAL3 genes confers increased levels of proline biosynthesis and the enhancement of salt tolerance in cultured tobacco cells. Journal of Experimental Botany 55:387-395.

Young C.C., Chang C.H., Chen L.F. y Chao C.C. 1998. Characterization of the nitrogen fixation and ferric phosphate solubilizing bacteria isolated from a Taiwan soil. Journal of the Chinese Agricultural Chemical Society 35:201-210.

Yun Y-S. y Volesky B. 2003. Modeling of lithium interference in cadmium biosorption. Environmental Science and Technology 37:3601-3608

Recibido: 17 de julio de 2007

Aceptado: 5 de abril de 2008
Zhang H.Y., Xu W.Z., Guo J.B., He Z.Y. y Ma M. 2005. Coordinated responses of phytochelatins and metallothioneins to heavy metals in garlic seedlings. Plant Science 169:1059-1065.

Zenk M.H. 1996. Heavy metal detoxification in higher plants: a review. Gene 179:21-30.

Zheng S.J., Ma J.F. y Matsumoto H. 1998. High aluminum resistance in buckwheat. 1. Al-induced specific secretion of oxalic acid from root tips. Plant Physiology 117:745-751.

Zhou J. y Goldsbrough P.B.1995. Structure, organization and expression of the metallothionein gene family in Arabidopsis. Molecular and General Genetics 248:318-328.

Zhu Y.G., Christie P. y Laidlaw A.S. 2001. Uptake of Zn by arbuscular mycorrhizal white clover from $\mathrm{Zn}$-contaminated soil. Chemosphere 42:193-1999. 